\title{
EAl EndorsedTransactions

\section{Deployment of free Wi-Fi voice communications to report crime incidents in Botswana}

\author{
A.W. Mogwe $e^{1, *}$ and K.K. Kenosi ${ }^{2}$ \\ ${ }^{1}$ Botho University. University of Botswana. P O Box 405749. Gaborone. Email: alphmog@gmail.com \\ ${ }^{2}$ Baisago University. Gaborone. Email: kennedykenosi@rocketmail.com
}

\begin{abstract}
The research explored the current scenario of crime in Botswana, and the current measures employed to help combat it, including usage of crime prevention clusters. Through usage of the quantitative approach, with the aid of questionnaire and interviews, the research found that, there is an increasingly growth of crime rate in Botswana, sustaining the literature findings. The research highlighted the presence of crime prevention clusters, but noted a disconnection between them and the police. The bleak state of communication between the two, and ineffectiveness of current measures, causes the disconnection. Cluster members have to use own limited resources to report crime to the police, and this disadvantages the crime prevention efforts. Ad-hoc networks with emphasizes on Wi-Fi networks were found to be a solution to the communication gap identified. In conclusion, the authors developed a Wi-Fi network facility model to enable cluster members to make voice calls with no costs.
\end{abstract}

Keywords: Ad-Hoc networks, Wi-Fi networks, Crime, Voice calls.

Received on 22 December 2017, accepted on 25 December 2017,published on 28 December 2017

Copyright (C) A.W.Mogwe and K.K. Kenosi., licensed to EAI. This is an open access article distributed under the terms of the Creative Commons Attribution licence (http://creativecommons.org/licenses/by/3.0/), which permits unlimited use, distribution and reproduction in any medium so long as the original work is properly cited.

doi: 10.4108/eai.28-12-2017.153523

\section{Introduction}

\subsection{Background and Motivation}

The advancement of Information and communications technologies (ICTs) has led to new innovations in the computing world, and benefited various sectors. It is very imperative to also use at our advantage the existing ICTs to help in the prevention and combating of crime, which has become a major concern within the borders of Botswana and outside. It is vital to also acknowledge the efforts by the police community whilst at the same time find alternative ways to help improve their efforts where there are shortfalls. Finding ways of infusing ICTs to combat and prevent crime will improve the police performance. With a variety of existing technologies, authors have found it fit to examine how the use of $\mathrm{Wi}-\mathrm{Fi}$ can benefit both the various communities in the areas of crime prevention, control and combating. The authors make available a description of the proposed Wi-Fi model which could be used to aid in communication to fight crime. Communication between various organs tasked with combating and preventing crime, is apparently one of the major bottlenecks to fighting crime, thus investigation on the Wi-Fi technology on how it can help ease the communication gap is carried within this research.

\subsection{Rise in crime a concern}

Crime is a major concern in every country both developing and developed. The challenge that developing countries face is lack of sufficient resources to fight crime. Criminals usually tend to get away with their criminal activities due to lack of real time responsive measures to apprehend them and bring them before the law. Even though countries are doing their best to equip their police departments, it has been evident that there is often a shortage of resources. Botswana as a developing country is faced with this predicament. There are also criminal gangs which form some organized groups

*Corresponding author. alphmog@gmail.com 
which are mandated with spying and forewarning each other of the position of the police.

The same problem is faced by South Africa which also one of the developing countries in Africa [1] and is believed to be advanced in crime fighting. Informal policing was introduced in South Africa to augment the formal policing [1] [2] [3]. Botswana just like South Africa and other developing countries, is not an exception to the rising crime situation, and stretched police resources, which has led to formation of crime fighting groups known as crime prevention clusters or cluster policing [4]. These groups are aimed at patrolling the neighbourhood to minimize any crime occurrences.

The groups are normally made up of unemployed people, especially the youth as a way of removing them from the streets and keeping them occupied by giving them a structured role in the society. Some of the main duties of these clusters are to patrol the streets in their neighbourhoods, to apprehend the culprits first hand and take them to the nearest police station and to inform the authorities when they see some activity that looks suspicious. To accomplish all this, the group members have to rely mainly on cellular phones to alert each other as well as the police about what is going on around them. The unemployment status of the members of the cluster groups leaves them with limited financial power and the inadequate government stipend is enough for their sustenance only.

This situation detrimentally impacts on the whole efforts of the government to fight crime [5]. For example according to the Botswana police crime statistics report, crimes such as motor vehicle, stock theft and rape attempts have seen an increase of $8.81 \%, 7.89 \%$ and $0.62 \%$ respectively [4]. Thus in the year 2013 murder cases escalated to 137 as compared to 116 in 2013, rape decreased from 993 in 2012 to 972 in 2013 with a slight 21 decrease, motor vehicle theft decreased from 200 in year 2012 to 180 in 2013 which was just a marginal 20 cases decrement and the only satisfactory decrement was seen on the armed robbery case as the crime decreased from 1058 in 2012 to 780 cases in 2013 [4]. Therefore, this crime situation [4], and the failure of crime clusters to combat crime effectively, research of how $\mathrm{Wi}-\mathrm{Fi}$ (a popular wireless technology) can be used to aid communication between members of crime prevention clusters and police was done through Wi-Fi. Wi-Fi is a versatile technology with capabilities of facilitating free to air cellular communications [6], and authors researched and designed a system to be used to combat crime by merging the communication gap between police and cluster groups, whilst ensuring that there is no violation of the rules of ad hoc and peer to peer communications or any commercial service agreements..

\section{Methodology}

Selection of a research paradigm is vital [11] [12] [13]. The research addressed the limitations faced by the Botswana crime prevention clusters to communicate well with each other and the police by developing a system to allow free voice call over a Wi-Fi network system which can enable them to execute their job effectively. The research made use of a deductive approach in order to answer the research questions in depth. Data from both primary and secondary research was considered to be vital. Secondary research data was acquired from studying existing literature from credible databases. Primary research data helped builds up a hypothesis from the responses given by the stake holders. Moreover quantitative research method was used. The Quantitative research methodology deals with the numerical aspect of research such as statistics. This methodology intends to answer questions such as how much, how often and any other question which may require any numeric justification. The quantitative methodology was used to establish the current Botswana crime statistics in places where the crime prevention clusters are deployed. Questionnaire and interview techniques were also used as part of the study to gather information.

\section{Voice over IP via BLUETOOTH/Wi-Fi peer to peer}

The use of telephony services through Global Systems for Mobile (GSM) and Third Generation mobile communication has proven to be very costly to users [6] [7]. Two principle methods can be used to reduce telephony costs through implementing IP over telephony [6] [8]. One of the suggested methods is the utilization of Wi-Fi over access points (AP) and the other one is the use of Wi-Fi over peer to peer (P2P) networks [6]. Both these approaches are aimed at providing telephone services at no cost to the users [6] [7]. Therefore instead of users registering to a particular centralized database service for billing purposes, an algorithm capable of mapping each mobile number to a particular unique Internet Protocol (IP) address can be developed [6]. Once the mobile numbers are mapped to their IP addresses then a peer to peer connection is established between the communicating devices for as long as they are running the same algorithm.

A server that provides the Dynamic Host Configuration Protocol (DHCP) is used. The DHCP is responsible for dynamically and automatically allocating IP addresses to the IP phones. To accomplish all these, the Voice over Internet Protocol (VoIP) technology is used for transferring voice signals from the source to the destination [6]. Devices searches for each other on the same Wi-Fi range and if they find each other they make use of the access points or peer to peer $(\mathrm{P} 2 \mathrm{P})$ with conversion of mobile number to an IP address done to establish a connection. According to Sundar et al [6] the system utilizes the mobile garget phone book for the conversion of phone numbers to IP addresses. Through this system users can send and receive Short Message Services (SMS) and Multimedia Messaging Service (MMS) as well as the utilization of outbox and inbox facilities. For the mobile phones to operate they have to identify each other on the WiFi or Bluetooth network [6] and if the phone is reachable by the caller then a peer to peer connection is established between the phones and voice data is exchanged between them. Wi-Fi over Peer to Peer (P2P) or Wi-Fi over Access Point (AP) can be used together with a novel algorithm to 
establish a no cost wireless phone communication [7], sustaining previous findings [6].

\section{Results Findings and Analysis}

(i) Questions on "Has Crime increased over the years" and "Rate the crime prevalence rate"

Respondents indicated that crime has increased over the years with $33.3 \%$ strongly agree, $26.7 \%$ agreeing with a compounded total of $60 \%$. There were some respondents that disagreed the crime rate was increasing. A total of $6.7 \%$ strongly disagreed and $13.3 \%$ disagreed respectively hence when compounded it goes to $20.0 \%$ with the not certain of the crime situation standing at $20 \%$. In addition, this tallies with crime prevalence rate which shows to be high. Majority of the respondents have chosen to say crime prevalence is high at a combined of $80.0 \%$ of the very high $(10.0 \%)$ and high (70\%) components, with those indicating that the crime rate is low standing at $6.7 \%$ and those not certain standing at $13.3 \%$ sustaining the secondary findings [4].

\section{(ii) Common criminal activities}

The respondents were asked to indicate the commonality of certain crimes outlined as stealing, robbery, rape, murder, manslaughter and fraud. From the feedback, it can be noted that a combined $86.7 \%$ agree that stealing is common crime in Botswana. The combined agree component is made up of the $30.0 \%$ of strongly agree and $56.7 \%$ of the agree respondents leading to a total of $86.7 \%$. Respondents on robbery stands at a combined $66.7 \%$ that robbery is common. In addition, respondents also agree that rape is common at $66.7 \%$. Murder is another criminal activity which has been deemed to be common in Botswana with $46.7 \%$ of combined respondents who agree that it is common. Moreover, respondents also agreed that manslaughter is common at $50.0 \%$. As for the fraud criminal activity, it can be noted that a combined of $86.7 \%$ agree that fraud is common in Botswana. All the findings sustain secondary findings [4].

\section{(iii) Are current measures effective}

Current measures to combat crime have been found to be ineffective, as crime continues to rise. Respondents disagree at a combined percentage of $66.7 \%$ that the current measures are not effective. Therefore, respondents have noted that crime clusters end up not actively participating in combating crime with $56.7 \%$ noting that. Their lack of full participation is noted due to lack of communication tools, with $73.3 \%$ of respondents noting lack of communication tools as a hindrance. Majority of the respondents at $80.0 \%$ agree that lack of crime fighting equipment is a hindrance to cluster participation. All this sustains the findings on crime in Botswana [4].

(iv) Internet and Wi-Fi usage and feasibility for usage
The question on this aspect sought to understand internet availability and usage in the police departments and if the police departments will embrace utilisation of $\mathrm{Wi}-\mathrm{Fi}$ in their environments. Respondents $63.3 \%$ noted that there is no internet connectivity in the police stations. Though the police environments had limited or lack internet connection, majority at $63.3 \%$ noted that it would be feasible to use $\mathrm{Wi}$ Fi resources in the station, thus they welcomed utilisation of Wi-Fi. This shows that lack of resources is an impediment to many initiatives, and many respondents are willing to try new ways of fighting crime.

\section{The System}

\subsection{Implementation}

The Botswana Police crime prevention cluster members are provided with android Samsung smart phones equipped with the CSipSimple software. CSipSimple application allows users to make free VoIP calls by taking advantage of the available Wi-Fi network infrastructure. Two $\mathrm{P} 2 \mathrm{P}$ radio antennae where mounted to the already existing Botswana telecommunication towers. The antennae are then linked to Cisco outdoor industrial access points which then transmit to the user equipment which in this case is the user smart phones. The crime prevention cluster members' phones are installed with CSipSimple and their registration information configured on the application as required. This includes information such as Passwords, Caller IDs, Usernames and Account names as well as the server. CSipSimple offers the user an option to either make a call through Global System for Mobile communication (GSM) or Wi-Fi. Once the user selects GSM then a normal cellular call is established and the user has to incur the charge rates. If the user selects Wi-Fi then a VoIP over Wi-Fi call is established at no charge.

Cisco Aironet 1570 series outdoor access points are mounted at the top of public buildings such as post offices, Clinics so as to transmit wireless signals to users. All the access points are registered with the wireless controller at the police station for centralized management. To avoid the public abusing the wireless network the access points (Service Set Identifier) SSID broadcasting are disabled and (Media Access Control) MAC address filter enabled. The WiFi network is used only for voice calls and not for internet access. A router is used to block any web based traffic which may be transmitted on the network. A VoIP Private Branch Exchange (PBX) is located at the police station to establish wireless voice calls between the mobile phone users over $\mathrm{Wi}$ Fi.

\subsection{Logical diagram}

The access points are controlled by the wireless controller at the police station. For security reasons to avoid intruders taping into the wireless network the (Zimmermann RealTime Transport Protocol) ZRTP protocol is used to encrypt the voice calls. Whenever a user makes a call the call is 
forwarded to the PBX server through the access point. The PBX initializes a call session between the communicating phones. Access points normally have limitations when it comes to the distance covered as well as prone to obstruction. Radio antennae mounted on the Botswana telecommunication Corporation towers are responsible for covering distances which are more than 1 kilometer then relay the signal transmission to (Wireless Local Area Network) WLAN access points. Users then receive the Wi-Fi signal from the access point.

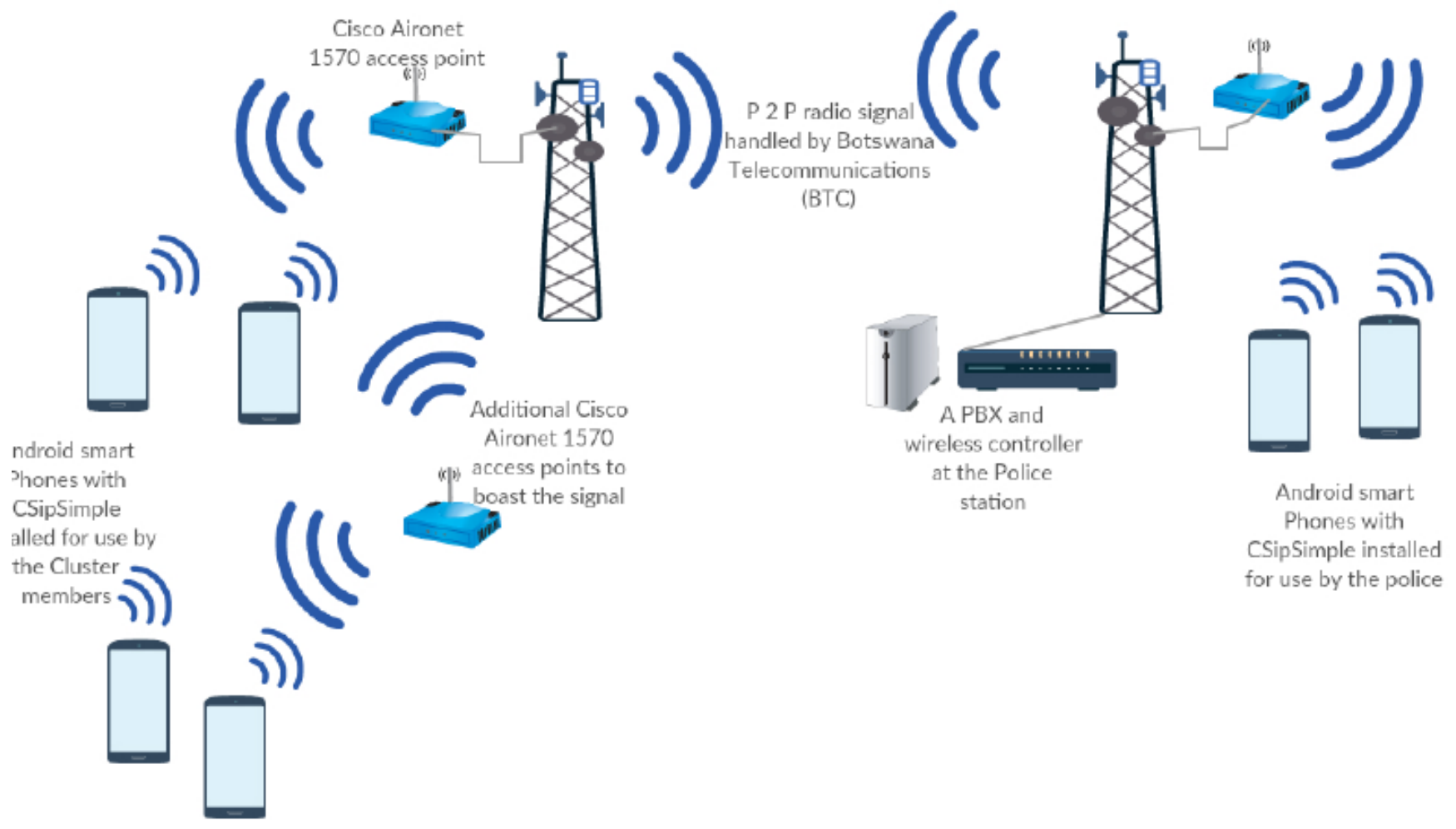

Figure 1. The Model Proposed by authors

According to Kbar et al [7] Global System for Mobile (GSM) and 3rd Generation telecommunication are very costly to costly to customers. The above system in Figure 1 depends on the distributed architecture where users are not going to register their information to any server services for billing [7] thus reducing the cost to nil due to it using Wi-Fi technology. To proactively prevent the possibility of IP address conflicts IP version 6 (IPv6) was used to address the communicating devices instead of IP version 4(IPv4) which comprises of fewer IP addresses and it has been exhausted. IPv6 addresses are 128 bits long and can accommodate millions of unique usable IP addresses as compared to IPv4 which is 32 bits long and accommodating less IP addresses [14]. Moreover, as stated [7], the area code and the phone number are in the 0-9 format which means they can be given any number between 0 and 9 .
The DHCP will be used to automatically and dynamically allocate unique IP addresses to mobile phones. This will cause the novel algorithm to convert each mobile number to a unique IP address, then the mapped addressed do not have to be stored because a new unique mapping is going to take place every time a call is made. The mobile phones will be installed with the novel algorithm application for those intending to communicate using voice over IP through WI-FI as this conversion is a two way process

Whenever, any mobile phone intends to establish a call to another, a conversion of both IP mobile numbers have to be converted to unique IPv6 IP addresses. Once the IP addresses have been successfully converted, a peer to peer connection through the use of the mapped IP addresses to phone numbers is made. For as long as the two mobile phones exist in the same $\mathrm{Wi}-\mathrm{Fi}$ range then IP for the calling phone will be converted back to its phone number and display on the receiving mobile phone leading to it to ring 
after a virtual connection has been established. For the communication to take place the receiving mobile phone has to accept connection. In the case that the mobile phones used are not in the same Wi-Fi range, then the calling mobile phone establishes connection to nearby existing access point (AP) to establish a virtual link between them. Both these processes use voice over IP (VOIP) technology [7]. Accordingly, if it happens that the mobile devices fail to identify each other on any Wi-Fi range then the user will be prompted by a message giving them an option of establishing a voice call through the traditional costly GSM platform.

\section{Conclusion}

The authors conclude that the research was of higher significance. Through research carried, crime situation in Botswana is high and growing with measures employed being not effective enough to combat the crime situation. Moreover, there is presence of crime cluster groups but they are not fully equipped. Thus, utilisation of Wi-Fi technology can help ease the communication gap. There is enough evidence that this is a feasible project which if implemented carefully and diligently could be beneficial for Botswana.

The authors developed a Wi-Fi network facility model to enable cluster members to make voice calls with no costs, following the literature review which sustained that it is possible to provide uncharged voice calls bypassing the GSM, 3G as well as $4 \mathrm{G}$ networks using Wi-Fi technologies or peer to peer networks. Through the research carried, this shows that utilisation of Ad-hoc networks with emphasizes on Wi-Fi networks, were found to be a solution to the communication gap identified and increasingly play an important role in the communication setup nowadays. Nonetheless, extensive research should be done and appropriate $\mathrm{Wi}-\mathrm{Fi}$ technologies selected for deployment with appropriate communication devices such as android mobile phones rolled out and given to crime prevention cluster participants who will be part of the WiFi systems being utilised. More research is needed with emphasis on designing and development of algorithms which are of suitable means in combating crime using Wi$\mathrm{Fi}$ and also security.

\section{Acknowledgements}

We would like to thank our families for their continued support, the participants who took part in this study, Mogoditshane Police Station and all those who have contributed to the study.

\section{References}

[1] Manaliyo, J.C. (2016). Barriers to community participation in crime Prevention in low income communities in Cape Town. International Journal of Social Sciences and Humanity Studies. Vol (8), no 1, 2016
[2] Kornhauser, RR 1978, Social Sources of Delinquency: An Appraisal of Analytic Models, University of Chicago Press.

[3] Benson, BL. (1989). Enforcement of Private Property Rights in Primitive societies:Lawwithou Government. The journal of Libartarian studies.ix (1), 14-20.

[4] Botswana police weekly crime report http://www.police.gov.bw/bps-content.php?cid=861 (Retrieved 6 April 2015)

[5] Matambo, K. (2015). Budget Speech. Available from http://www.parliament.gov.bw/phocadownload/ContentArc hives/Budget-Speech/2015budgetspeech.pdf (Retrieved 4 April 2015)

[6] Sundar, D.C.E. Krishna Kumar, M. Selvinpremkumar, P. Chinnadurai , M. (2012) Voice over ip via bluetooth/wi-fi peer to peer. IEEE-International Conference On Advances In Engineering, Science And Management (ICAESM 2012)

[7] Kbar, G. Mansoor, W. Naim, A. (2010) Voice over IP mobile telephony using WIFI P2P. 2010 Sixth International Conference on Wireless and Mobile Communications

[8] Patel, J.J. (2014) IP-Based Connection Between Mobile Phones A Novel Connection System Of Mobile Phones In A Wireless Network

2014InternationalConferenceonComputerCommunicationa ndInformatics(ICCCI2014

[9] Africa check 2014, https://africacheck.org/factsheets/factsheet-south-africasofficial-crime-statistics-for-201314/ (accessed 24th August 2015)

[10] Bank of Botswana 2015, http://www.bankofbotswana.bw/indicators/exchanges(Acce ssed 6 April 2015)

[11] Dash.NK. (2005). Selection of the Research Paradigm and Methodology. Available:

http://www.celt.mmu.ac.uk/researchmethods/Modules/Sele ction of methodology/. Last accessed 23th August 2015.

[12] Gable, GG. (1994) Integrating case study and survey research methods: an example in information systems. European Journal of Information Systems 3(2):pp. 112-126

[13] Yates, S.J. (2004) Doing Social Science Research, London, Sage Publications/Milton Keynes, The Open University http://www.academia.edu/7873147/Community_Participati on_in_Crime_Prevention_Informal_Social_Control_Practic es_in_Site_B_Khayelitsha_Township)

[14] Teare, D., Vachon, B., Graziani, R., Froom, R., Frahim, E., Ranjbar, A. (2012). CCNP Routing and Switching.

Foundation learning guide library.

[15] J. Hu, I. Khalil, S. Han, and A. Mahmood, "Seamless integration of dependability and security concepts in SOA: A feedback control system based framework and taxonomy," Journal of Network and Computer Applications, Volume 34, Issue 4, July 2011, Pages 11501159.

[16] B. Tian, S. Han, S. Parvin, J. Hu, and S. Das, "Self-healing key distribution schemes for wireless networks: A survey," Computer Journal, Volume 54, Issue 4, April 2011, Pages 549-569.

[17] X.D. Hoang, J. Hu, and P. Bertok, “A program-based anomaly intrusion detection scheme using multiple detection engines and fuzzy inference," Journal of Network and Computer Applications, Volume 32, Issue 6, November 2009, Pages 1219-1228. 
[18] J. Zhang, Y. Xiang, Y. Wang, W. L. Zhou, Y. Xiang, and Y. Guan, "Network Traffic Classification Using Correlation Information," Ieee Transactions on Parallel and Distributed Systems, vol. 24, no. 1, pp. 104-117, Jan, 2013.

[19] Y. Xiang, K. Li, and W. L. Zhou, "Low-Rate DDoS Attacks Detection and Traceback by Using New Information Metrics," Ieee Transactions on Information Forensics and Security, vol. 6, no. 2, pp. 426-437, Jun, 2011.

[20] J. Zhang, X. Chen, Y. Xiang, W. L. Zhou, and J. Wu, "Robust Network Traffic Classification," Ieee-Acm Transactions on Networking, vol. 23, no. 4, pp. 1257-1270, Aug, 2015.
[21] H Wang, J Cao, Y Zhang, A flexible payment scheme and its role-based access control.

[22] IEEE Transactions on knowledge and Data Engineering 17 (3), 425-436, 2005.

[23] X Sun, H Wang, J Li, TM Truta. Enhanced p-sensitive kanonymity models for privacy preserving data publishing. Transactions on Data Privacy 1 (2), 53-66, 2008

[24] H Wang, Y Zhang, Untraceable off-line electronic cash flow in e-commerce, Australian Computer Science Communications 23 (1), 191-198, 2001 